\title{
论 文薄膜基荧光传感专刊
}

\section{柔性PDA薄膜阵列用于双模光学检测VOC标志物 气体}

徐华”, 安远, 张明坤, 李佳佳, 顾忠泽*

东南大学生物科学与医学工程学院, 生物电子学国家重点实验室, 南京 210096

*通讯作者, E-mail: huaxu@seu.edu.cn; gu@seu.edu.cn

收稿日期：2019-06-20; 接受日期：2019-07-19; 网络版发表日期：2019-09-24

国家自然科学基金(编号: 51573025, 21103020)、江苏省重点研发计划(编号: BE2016002-1)和中央高校基本科研业务费资助项目

摘要本研究以亲油性的双面胶作为基底, 利用滴涂二乙炔单体结合紫外光聚合来制备均匀的聚二乙㶧(PDA) 薄膜，通过荧光和颜色两种信号变化模式(即“双模光学检测”)研究了PDA薄膜对VOC气体的响应性，发现制备的 PDA薄膜在 $2 \mathrm{~min}$ 内就可以实现明显的荧光和颜色变化, 有效解决了目前PDA薄膜在VOC气体检测方面存在响应 速度慢、薄膜均一性差等问题。此外, 为解决单一PDA薄膜的交叉响应性问题, 本研究制备了四种不同的基于双 面胶基底的PDA薄膜，并将制备的4种PDA薄膜集成到一片PDMS薄膜基底上来构建柔性的传感阵列，利用阵列 的颜色变化结合模式识别技术，实现了对 8 种 $\mathrm{VOC}$ 气体的快速、灵敏区分. 进一步将制备的PDA薄膜阵列用于健 康人、模拟糖尿病及肾病患者呼出气体中 VOC标志物的辨别和分析研究，发现可以将三类人的呼出气体清晰地 区分，说明了该阵列在呼气疾病诊断中的应用前景。与目前报道的PDA薄膜阵列相比，本研究中基于双面胶基底 的PDA薄膜阵列具有气体响应速度快、灵敏性高、柔韧性好、制备工艺简单、成本低、易于大规模制备等优 点, 有望用于实际VOC气体检测研究中.

关键词 PDA薄膜阵列, $\mathrm{VOC} 气$ 体检测, 荧光, 颜色, 疾病诊断

\section{1 引言}

快速、准确地检测挥发性有机化合物(volatile organic compounds, VOCs)气体在爆炸物、毒品、有机 污染物检测以及重大疾病早期诊断等领域都具有广阔 的应用前景 ${ }^{[1 ~ 3]}$. 尤其是近年来，随着现代医学诊断技 术的不断发展, 研究人员发现人体呼出气体中VOCs气 体的组成和含量分布与一些代谢性疾病密切相关，如 糖尿病患者呼气中的丙酮含量会明显增大。这些
VOCs气体可以看作是这些疾病的标志性气体，通过 检测呼气中的这些VOCs气体变化就可以实现对这些 疾病的快速判断 ${ }^{[4-7]}$. 该技术具有操作简便、检测速度 快且对人安全无创等特点, 在疾病早期诊断方面极具 应用前景. 实现其应用的关键是开发高性能、便携式 的VOC标志物气体检测系统.

聚二乙炔 $(\mathrm{PDA})$ 是一种具有 $\pi$ 共轭结构的线型聚 合物大分子，其由二乙炔单体自组装后经 $254 \mathrm{~nm}$ 的紫 外光照聚合而制备. 一般聚合得到的PDA都呈现蓝色,

引用格式: Xu H, An Y, Zhang M, Li J, Gu Z. Flexible PDA film array for dual-mode optical sensing of VOC biomarker vapor. Sci Sin Chim, 2020, 50: 135-142, doi: 10.1360/SSC-2019-0072 
并且没有苂光. 这种蓝色的PDA在外界刺激下会发生 结构转变, 产生明显的颜色变化, 典型的是变为红色, 并且红色的聚二乙炔会出现明显的荧光发射. 因此, 各 种基于PDA的色敏及苂光传感器已被广泛开发用于气 体小分子、生物大分子、溶剂、热、金属离子等传感 检测研究 ${ }^{[8 \sim 10]}$. PDA聚合物分子由于具有 $\pi$ 共轭结构很 容易聚集在一起, 限制了其均一薄膜的构建. 为了解决 这一问题, 同时实现PDA传感器使用的便携性和稳定 性，通常将PDA固定在基底表面来制备PDA薄膜传感 器. 目前, 玻璃、二氧化硅、琼脂糖、藻酸钠纤维等 都已经被用于PDA的基底来构建薄膜传感器 ${ }^{[11 ~ 15]}$. 最 近的研究显示, 固体基底不仅可以影响二乙炔单体的 聚合行为，还可以提高PDA薄膜检测目标分析物的灵 敏性 ${ }^{[15]}$.

柔性可穿戴传感器体积小、重量轻, 因其可以直 接贴附或穿在人们身上或者衣服上连续监测人体的生 理状态而受到广泛关注。各种基于柔性基底材料的 PDA薄膜传感器已被构建用于VOC标志物气体检测 研究. Wang 等 ${ }^{[16]}$ 通过在柔性聚对苯二甲酸乙二酯 (PET) 基底上聚合含二硫化钼的二乙炔来构建柔性 PDA薄膜传感器, 可直接戴在人体手腕上, 通过PDA薄 膜明显的颜色变化来检测 $N, N$-二甲基甲酰胺气体. Wang等 ${ }^{[17]}$ 也报道了通过在PET基底上构建含石墨烯 的PDA薄膜传感器, 利用PDA薄膜的荧光和颜色变化 实现了对多种不同的 $\mathrm{VOC}$ 气体(如 $N, N$-二甲基甲酰 胺、甲醇、三氯甲烷、四氢呋喃等)的检测研究. 由于 单一的PDA薄膜传感器普遍存在着交叉敏感，往往会 对多种被测气体响应，因而很难有选择地测量出某种 特定气体的成分和含量. 为了解决这一问题，有研究 者报道了利用柔性的滤纸作为基底，通过在其表面不 同的位置分别滴加 4 种不同的二乙炔单体，以光聚合 法制备了基于 4 种不同PDA薄膜的柔性传感阵列. 通 过PDA薄膜阵列的荧光和颜色变化，结合模式识别技 术，实现了对 18 种不同VOC气体的高灵敏区分 ${ }^{[18]}$. 此 外，电纺纳米纤维也被用于柔性基底来构建PDA薄膜 传感阵列. 通过分别共纺不同的二乙炔单体和聚环氧 乙烷(PEO)的混合物, 再利用光聚合来法可制备PDA/ PEO的复合传感薄膜 ${ }^{[19]}$. 通过三个不同PDA/PEO薄膜 阵列的苂光和颜色变化, 结合模式识别技术, 实现了对 7种有机氨气体的高灵敏区分. 尽管目前这些传感阵列 已经实现了对多种 VOC气体的灵敏检测，但其仍存在
着响应速度慢、PDA薄膜均一性及稳定性差等问题. 如已报道的基于滤纸和纳米纤维膜的PDA阵列检测 VOC气体的响应时间通常都在 $1 \mathrm{~h}$ 以上; 其次，由于滤 纸的主要成分是纤维素, 与水有很强的亲和性, 而与有 机化合物的亲和力很弱. 二乙炔单体溶液在滤纸表面 干燥过程中，很容易聚集在一起，导致制备的PDA薄 膜均一性差，并且制备的PDA薄膜在滤纸表面的稳定 性也较差，很容易脱落下来. 利用共纺法可以实现基 于纳米纤维PDA薄膜的均匀、稳定制备，但其制备工 艺复杂、制备时间较长. 这些都限制了 PDA薄膜阵列 在VOC气体检测方面的发展和实际应用，因此需要开 发新型的PDA薄膜阵列来解决这些问题.

在本研究中，我们提出利用具有强亲油性的双面 胶作为基底来构建新型的PDA薄膜阵列用于 $\mathrm{VOC}$ 气 体的传感检测研究. 我们利用滴涂结合光聚合法在双 面胶基底上构建了均匀的PDA薄膜，通过荧光光谱和 颜色变化研究了其对 $\mathrm{VOC} 气$ 体的响应性, 发现制备的 PDA薄膜在 $2 \mathrm{~min}$ 内可以实现明显的颜色和苂光光谱 变化. 我们进一步利用四种不同的基于双面胶基底的 PDA薄膜并将其集成在一片PDMS基底上来构建传感 阵列, 利用薄膜阵列的颜色变化结合模式识别技术, 实 现了对 8 种 $\mathrm{VOC}$ 气体的快速、灵敏区分. 我们还将制 备的PDA薄膜阵列用于健康人、模拟糖尿病患者及肾 病患者呼出气体中VOC标志物的辨别和分析研究，发 现其可以将三类人的呼出气体清晰地区分. 与目前报 道的PDA薄膜阵列相比，本研究基于双面胶基底的 PDA薄膜阵列具有气体响应速度快、灵敏性高、柔韧 性好、制备工艺简单、易于大规模制备等优点，有望 实际用于VOC气体检测分析中.

\section{2 实验部分}

\section{1 试剂与仪器}

所有的有机溶剂均为分析纯并购于国药集团化学 试剂有限公司(中国); 二乙炔单体：10,12-十七碳二炔 酸、10,12-二十二碳二炔二酸、10,12-二十五碳二炔 酸和 10,12-二十三碳二炔酸购于北京伊诺凯科技有限 公司; 双面胶购于 $3 \mathrm{M} 中$ 国有限公司.

扫描电子显微镜(Renishaw公司, $6500 \mathrm{~F}$, 英国); 本 实验所有的荧光图像均通过倒置荧光显微镜(奥林巴 斯，IX71)测量; 苂光光谱通过带有光纤光谱仪的苂光 
光学显微镜测量.

\section{2 实验方法}

(1) PDA薄膜阵列的制备. 取 $10 \mathrm{mg}$ 二炔单体加入 $1 \mathrm{~mL} \mathrm{~N}, N$-二甲基甲酰胺(DMF)中溶解分散, 得到的溶 液通过滤膜除去已经聚合的分子, 得到了无色的二乙 炔单体溶液. 以商品化双面胶作为基底, 在其表面滴 涂配制的二乙炔单体溶液，每 $1 \mathrm{~cm} \times 1 \mathrm{~cm}$ 的区域使用 $50 \mu \mathrm{L}$ 单体溶液. 将其放置于暗处干燥 $48 \mathrm{~h}$, 使 DMF完 全挥发. 用波长 $254 \mathrm{~nm}$ 紫外灯照射 $1 \mathrm{~min}$, 聚合生成 PDA薄膜.

四种二乙炔单体：10,12-十七碳二炔酸、10,12-二 十二碳二缺二酸、10,12-二十五碳二炔酸、10,12-二 十三碳二炔酸分别滴涂在双面胶基底上经 $254 \mathrm{~nm}$ 光照 制备了四种PDA薄膜.

聚二甲基硅氧烷(PDMS)薄膜按照以前报道的方 法制备 ${ }^{[20]}$ : 将PDMS预聚体和交联剂按 10:1 $(w / w)$ 的比 例均匀混合, 充分摚拌形成均匀的预聚物, 真空脱气后 倒入培养血中, $80^{\circ} \mathrm{C}$ 加热交联得到PDMS薄膜.

将双面胶没有PDA的一面粘在PDMS基底上，将 制备的四种PDA薄膜都粘到一片PDMS薄膜上，就得 到柔性的PDA薄膜阵列.

(2) VOC气体检测. 在室温下, 在一个玻璃血中加 入少量待测VOC气体的溶液, 密闭容器中保持 $30 \mathrm{~min}$. 然后将聚二乙炔薄膜放入该密闭容器中, 保持 $10 \mathrm{~min}$ 后取出, 观察薄膜的苂光光谱或颜色变化. 我们选取 了四氢呋喃、丙酮、苯、甲苯、甲醇、乙醇、三氯甲 烷、氨气等 8 种VOC气体测试.

(3) 实验数据分析. 聚类分析(HCA)和主成分分析 (PCA)的结果分别通过IBM SPSS Statistical 21和Social Science 13软件包统计程序进行.

\section{3 结果与讨论}

\section{1 基于双面胶基底的PDA薄膜制备及VOC气体 检测性能研究}

本研究利用具有强亲油性的双面胶作为基底来构 建新型的PDA薄膜传感器. 双面胶的主要成分是聚丙 烯酸酯，聚丙烯酸酯是一种高吸油树脂，对有机化合 物具有很好的亲和性，具有吸油速率快、保油能力 强、吸油种类多等特点 ${ }^{[21]}$. 利用双面胶作为基底, 可
以实现亲油性的二乙炔单体在其表面的均匀分布，提 高制备的PDA薄膜的均一性和稳定性; $\mathrm{VOC}$ 气体也具 有强的亲油性, 因此能够被双面胶快速吸附, 从而提高 $\mathrm{VOC}$ 气体在其表面的吸附速度、吸附量及响应时间, 实现对 VOC气体的快速、灵敏检测.

我们通过在双面胶表面上均匀滴涂10,12-二十五 碳二炔酸单体, 干燥后用 $254 \mathrm{~nm}$ 紫外灯光照引发二炔 酸单体聚合, 生成PDA薄膜. 二炔酸单体在干燥过程 中会自发组装形成有序晶体结构, 紫外光照时进行拓 扑化学聚合(图1(a)). 如图1(b)所示, 二炔酸单体在双 面胶基底上呈现白色, 聚合过程由于其结构发生拓扑 变化, 生成的薄膜变为明显的蓝色. 这一结果和以前 文献报道的结果是一致的 ${ }^{[17]}$, 也说明二乙炔单体已成 功聚合生成PDA薄膜.

图2显示了在双面胶基底上构建的PDA薄膜的 SEM图. 可以看出, PDA均匀、平整地分散在双面胶表 面，说明亲油性的双面胶可以实现PDA在其表面的均 匀分布.

我们将所制备基于双面胶基底的PDA薄膜放置于 四氢呋喃蒸气中测试其响应性能, 发现其在四氢呋喃 饱和蒸气中 $2 \mathrm{~min}$ 就发生了明显的颜色变化, 由蓝色变 化为红色, 如图3(a)所示. 此外, 蓝色的PDA薄膜是没

(a)

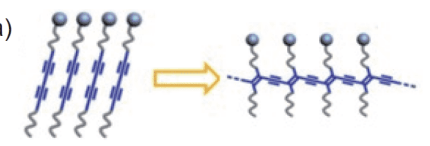

(b)

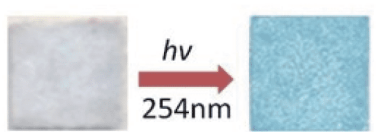

图 1 (a) 二乙炔单体聚合前后的结构变化; (b) 双面胶基底 上的二乙炔单体经光照后生成蓝色的PDA薄膜的照片(网络 版彩图)

Figure 1 (a) Structure change of diacetylene monomers before and after polymerization; (b) photographs of diacetylene monomers on the double-sides tape before and after UV-irradiation (color online).

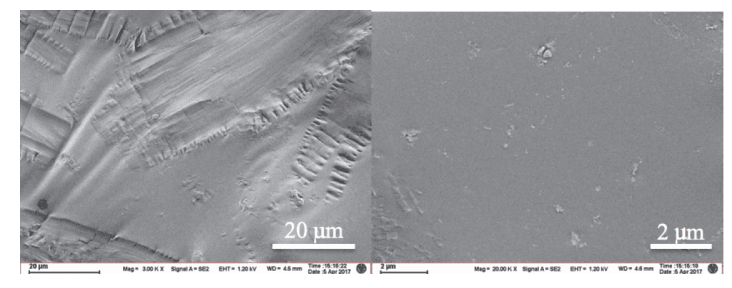

图 2 双面胶基底的PDA薄膜的SEM图

Figure 2 SEM images of the PDA film on the double-sides tape. 


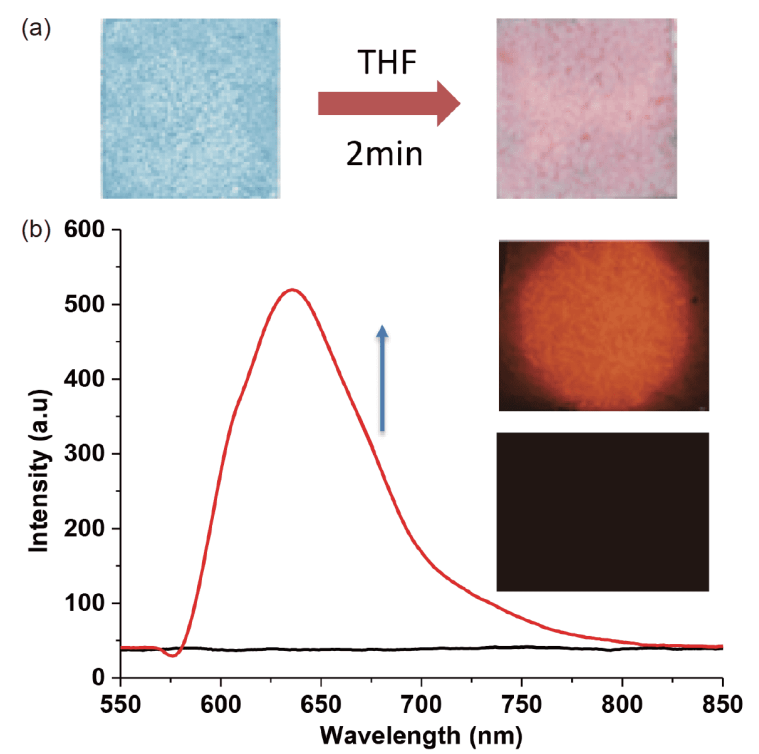

图 3 基于双面胶基底的PDA薄膜暴露在四氢呋喃饱和蒸 气中的响应性. (a) 颜色变化; (b) 苂光光谱及苂光颜色变化 (激发波长为 $490 \mathrm{~nm}$ ) (网络版彩图)

Figure 3 Response of the PDA film on the double-sides tape after exposure to saturated THF vapors. (a) Color change; (b) change of fluorescence spectra and fluorescence color (excitation $490 \mathrm{~nm}$ ) (color online).

有荧光的, 放置在四氢呋喃饱和蒸气中 $2 \mathrm{~min}$, 其苂光 照片呈现明显的红色苂光, 并且其苂光光谱在 $640 \mathrm{~nm}$ 呈现明显的吸收峰, 如图3(b)所示. 这一结果和以前文 献报道的结果是一致 ${ }^{[16 ~ 18]}$, 说明可以利用PDA薄膜的 颜色或荧光两种光学信号变化来检测四氢呋喃蒸气. 值得注意的是, 目前文献已报道的基于滤纸和纳米纤 维膜的PDA薄膜检测 $\mathrm{VOC}$ 气体的响应时间通常都在 $1 \mathrm{~h}$ 以上 ${ }^{[18,19]}$. 本研究开发制备的基于双面胶基底的 PDA薄膜 2 min 就可使四氢呋喃气体发生明显的颜色 或荧光变化. 这一结果表明利用油性的双面胶作为基 底可以大大提高PDA薄膜对 $\mathrm{VOC}$ 气体的响应速度, 降 低响应时间, 实现对 VOC气体的快速检测.

当 $\mathrm{VOC}$ 气体小分子进入PDA薄膜会影响到其主 链中的共轭结构 (重键), 即对其周围电子云分布有显 著影响, 从而改变外层电子跃迁的能级, 导致PDA薄 膜的光谱和颜色发生变化. 我们进一步利用以双面胶 为基底的PDA薄膜对四氢呋喃、苯、甲苯、三氯甲烷 四种VOC蒸气进行了测试, 发现其都呈现明显的颜色 和苂光变化, 如图4所示. 为了解决单一的PDA薄膜传 感器的交叉响应性问题, 我们又进一步开发制备了基 于双面胶基底的PDA薄膜阵列用于 $\mathrm{VOC}$ 蒸气监测

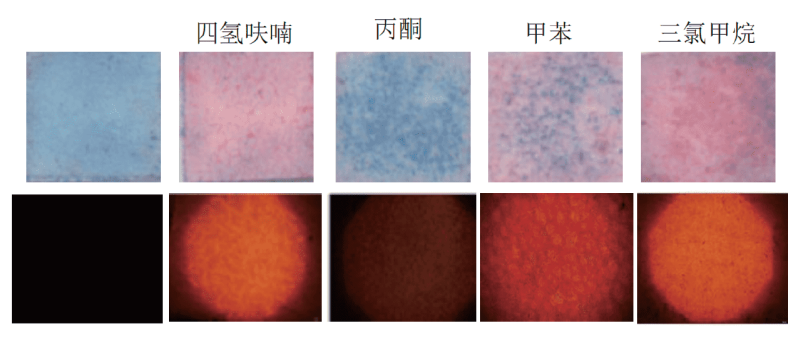

图 4 基于双面胶基底的PDA薄膜对不同 $\mathrm{VOC}$ 气体的响应 性. 上层: 颜色变化; 下层: 苂光颜色变化(网络版彩图)

Figure 4 Response of the PDA film on the double-sides tape after exposure to different saturated VOC vapors. Up: color change; down: fluorescence color change (excitation $490 \mathrm{~nm}$ ) (color online).

研究.

\section{2 基于双面胶基底的PDA薄膜阵列制备及VOC 气体检测性能研究}

已有的研究显示, 聚二炔主链发生非常微小的扭 转就可以很大程度地改变 $\pi$ 轨道的重叠, 使得吸收光 谱产生明显的蓝移. 由于聚二炔侧链与主链连接在一 起, 主链的扭转也会造成侧链构象的变化, 聚二炔颜 色的变化与主链、侧链都有着紧密的关系. 因此我们 选择四种不同的二炔单体聚合来构建PDA薄膜阵列. 这些聚二炔侧链构象不完全相同, 对外界刺激也会发 生不同的光学变化. 如图5(a)所示, 四种不同化学结构 的二炔单体在双面胶基底上聚合生成四种不同的PDA 薄膜, 进一步将这四种PDA固定在PDMS膜上, 实现柔 性PDA薄膜阵列的构建(图5(b)).

将制备的PDA薄膜阵列对四氢呋喃、丙酮、苯、 甲苯、甲醇、乙醇、三氯甲烷、氨气等 8 种 VOC蒸气 进行测试, 实验结果如图5(c)所示, 图中1、2、3、4分 别表示10,12-二十五碳二炔酸、10,12-二十三碳二炔 酸、10,12-二十二碳二缺二酸、10,12-十七碳二炔酸 聚合成的PDA薄膜. 可以看出, 阵列中每种PDA薄膜对 同一种 $\mathrm{VOC}$ 蒸气的颜色变化响应都是不同的. 因此, 阵列中四个PDA薄膜对同一种 $\mathrm{VOC}$ 蒸气的颜色变化 响应可以作为该气体的特征响应，根据这些不同的特 征响应模式就可以实现对不同VOC蒸气的区分. 如 图5所示, 根据PDA薄膜阵列的颜色变化, 就可以实现 对 8 种不同VOC蒸气的清楚区分.

此外, 我们还进一步研究了 PDA薄膜阵列检测 VOC气体的RGB数值(即红 $(\mathrm{R}) 、$ 绿 $(\mathrm{G})$ 、蓝 $(\mathrm{B})$ 三个颜 色通道)变化. 通过在每个PDA薄膜图像中随机选取 
$5 \times 5$ 像素的区域, 分别取其RGB数值的平均值, 每种气 体的测试结果各取 5 次，作误差线，计算每种气体RGB 数值的变化, 结果如图6所示. 可以看出, 每种气体都具 有独特的RGB数值变化值, 可以用来区分不同的VOC 气体. 此外, 根据PDA薄膜阵列的RGB数值变化, 不仅 不同化学类别的 $\mathrm{VOC} 气$ 体(如醇、酮、呋喃、苯等)可 以被区分，而且同一类VOC气体(如甲醇、乙醇; 苯、 甲苯)也可以通过PDA薄膜不同的RGB数值变化来区 分. 这一结果说明薄膜阵列在区别不同的 VOC气体上 具有很高的灵敏性.

为了研究所有样本之间的相似性, 计算所有样本 两两之间的平方欧氏距离，用相似度对样本空间进行
自然分组, 我们进一步通过聚类分析研究了 8 种 VOC 气体的检测结果. 如图7所示, 当取相似度为 1 时, 样本 可以被准确地区分为四氢呋喃、丙酮、苯、甲苯、甲 醇、乙醇、三氯甲烷、氨气，说明开发的PDA薄膜传 感阵列可以实现宽范围的 $\mathrm{VOC}$ 气体检测，具有优异的 可重复性和实用性.

\section{3 基于呼气中VOC标志物检测的疾病诊断研究}

最近的研究显示，在人体患有一些代谢性疾病的 状态下时，其呼出气中的 $\mathrm{VOC}$ 标志物的成分和浓度都 会发生变化. 例如，健康人呼出气体中丙酮的含量通 常是低于 $0.8 \mathrm{ppm}$, 而糖尿病患者呼出气体中丙酮的含 (a)<smiles>C=COCC</smiles>

(b)
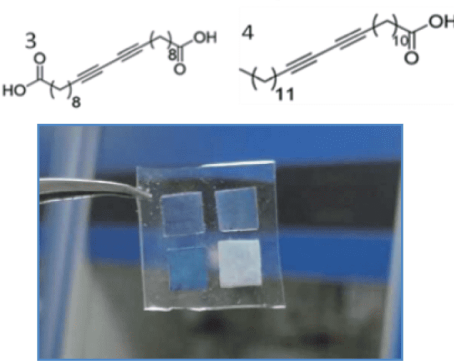

(c)

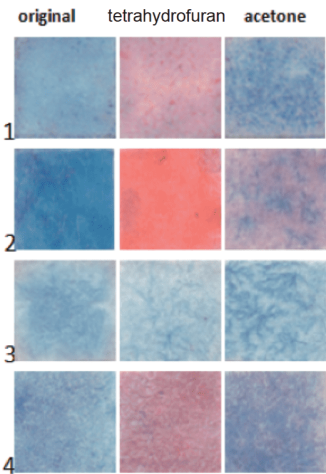

benzene

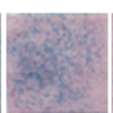

toluene methan
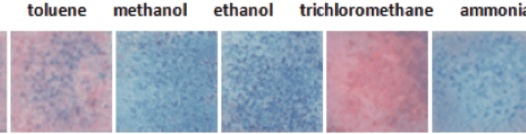

Fity
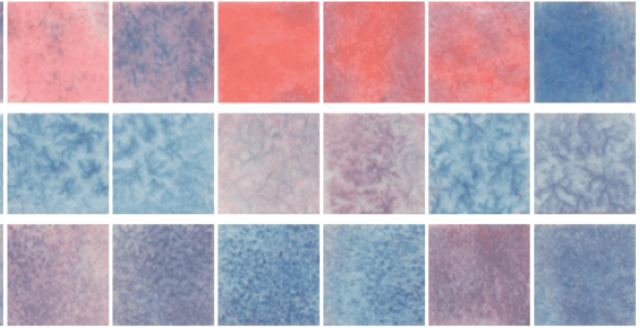

图 5 (a) 四种不同二乙炔单体的化学结构式; (b) 基于双面胶基底的PDA薄膜阵列照片; (c) PDA薄膜阵列检测 8 种不同VOC 蒸气的颜色响应性(VOC蒸气为 $298 \mathrm{~K}$ 下的饱和蒸气) (网络版彩图)

Figure 5 (a) Chemical structure formulas of four diacetylene monomers; (b) photograph of a PDA film array; (c) images of the PDA sensor array prepared from 1-4 exposed to various saturated VOC vapors (color online).

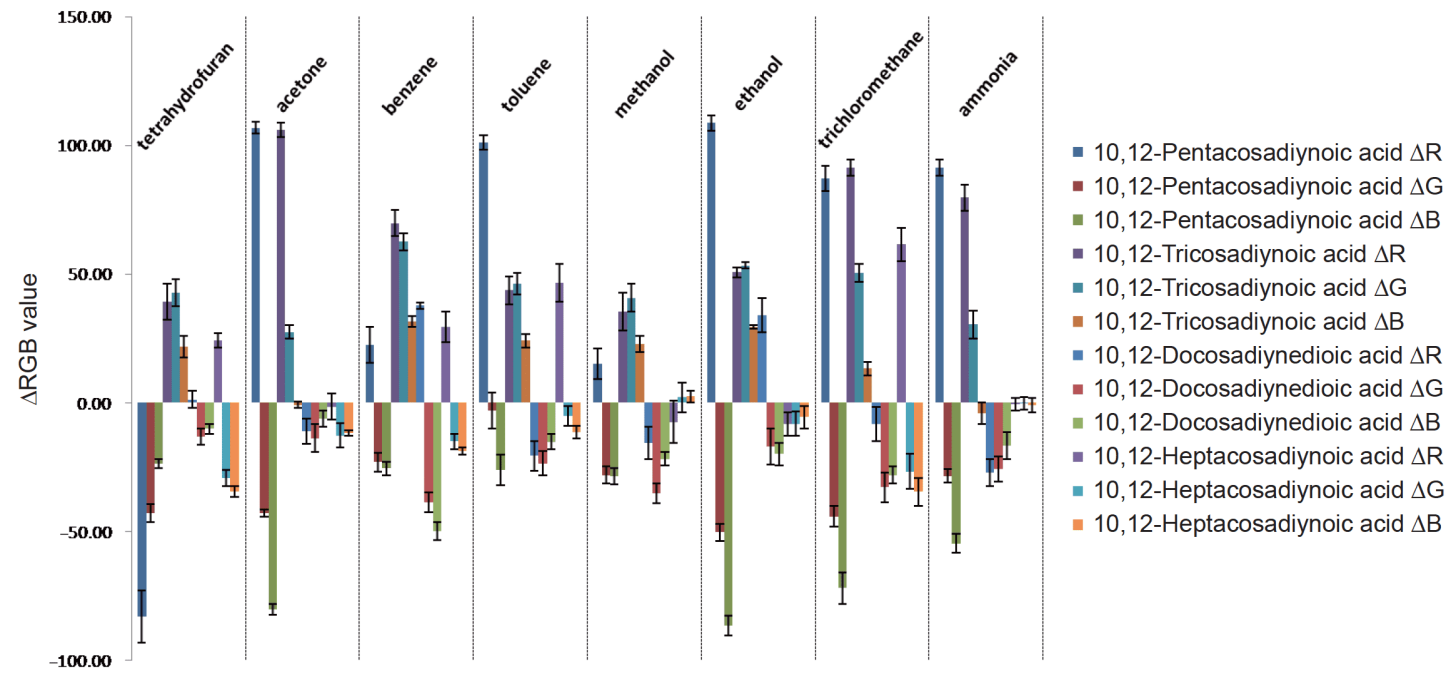

图 6 PDA薄膜阵列检测8种不同VOC蒸气的RGB值响应性(网络版彩图)

Figure 6 RGB color change profile of the PDA sensor array after exposure to saturated VOC vapors (color online). 


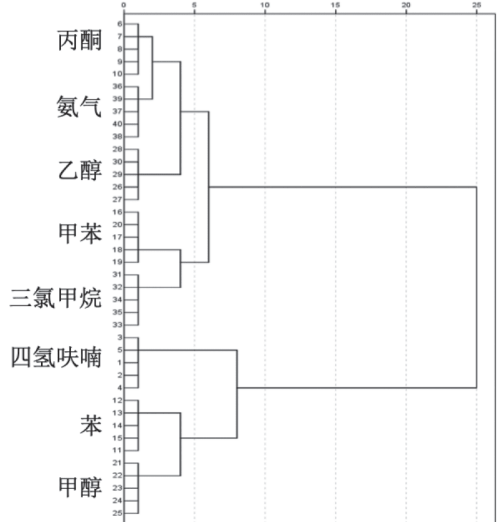

图 7 对 8 种不同VOC蒸气的聚类分析结果

Figure 7 Hierarchical clustering analysis (HCA) of eight VOC vapors.

量通常是高于 $1.8 \mathrm{ppm}^{[22 \sim 4]}$. 健康人呼出气体中氨气的 含量通常在400 1800 ppb，而终末肾病患者呼出气体 中氨气浓度可以达到 $2000 \mathrm{ppb}$ 以上 ${ }^{[25]}$. 我们通过将 $50 \mathrm{ppm}$ 丙酮和氨气分别引入健康人的呼出气当中，以 模拟糖尿病患者和肾病患者呼出的气体. 我们在 5 个 独立的实验中对 5 组呼气样本进行了测试，研究了我 们的PDA薄膜阵列对三种呼出气体(模拟糖尿病患者 呼出气、模拟肾病患者呼出气和健康个体的呼出气) 的响应, 读取PDA阵列在测试前后的RGB数值变化. 用 主成分分析方法对得到的数据进行分析, 结果如图8所 示. 从图中可以清楚地观察到三种呼出气样本之间的 区别，可以将5名健康个体、5名模拟糖尿病患者和 5 名模拟肾病患者的呼出气样本归类为三个没有重叠的 群集. 模拟测试结果说明我们的柔性PDA薄膜阵列在 VOC疾病标志物的检测方面具有潜在的应用价值.

\section{4 结论}

开发高性能、便携式、易于操作的 $\mathrm{VOC}$ 气体检 测系统在爆炸物、毒品、有机污染物检测以及重大疾

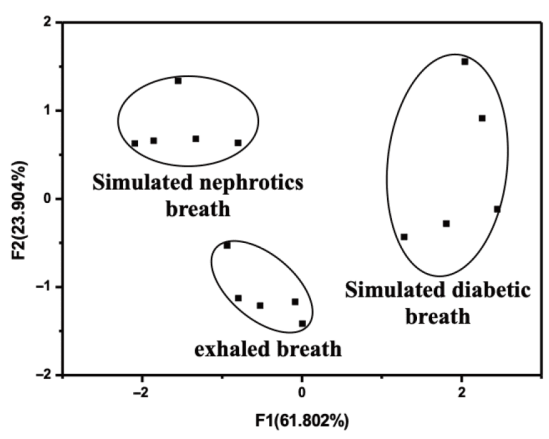

图 8 主成分分析健康人、模拟的糖尿病及模拟的肾病患 者的呼出气体结果

Figure 8 Principle component analysis (PCA) of exhaled breath of simulated nephrotic patients, diabetic patients, and healthy individuals.

病早期诊断等领域都具有重要的意义．本研究提出利 用具有强亲油性的双面胶作为基底来构建新型的PDA 薄膜阵列用于VOC气体的传感检测研究. 我们利用滴 涂结合光聚合法在双面胶基底上构建了均匀的PDA薄 膜，通过苂光光谱和颜色变化研究了其对 VOC气体的 响应性，发现制备的PDA薄膜在 $2 \mathrm{~min}$ 内可以实现明显 的颜色和荧光光谱变化，有效解决目前PDA薄膜响应 速度慢、PDA薄膜均一性差等问题. 我们进一步利用 四种不同的基于双面胶基底的PDA薄膜并将其集成在 一片PDMS基底上来构建传感阵列，利用薄膜阵列的 颜色变化结合模式识别技术，实现了对 8 种 $\mathrm{VOC}$ 气体 的快速、灵敏区分. 我们还将制备的PDA薄膜阵列用 于健康人、模拟糖尿病患者及肾病患者呼出气体中 VOC标志物的辨别和分析研究，发现其可以将三类人 的呼出气体清晰地区分, 说明该阵列在呼气疾病诊断 中具有良好的应用前景. 与目前报道的PDA薄膜阵列 相比，本研究中基于双面胶基底的PDA薄膜阵列具有 气体响应速度快、灵敏性高、柔韧性好、制备工艺简 单、易于大规模制备等优点，有望实际用于 $\mathrm{VOC} 气$ 体 检测分析中.

\section{参考文献}

1 Xu H, Wu P, Zhu C, Elbaz A, Gu ZZ. J Mater Chem C, 2013, 1: 6087-6098

2 Haick H, Broza YY, Mochalski P, Ruzsanyi V, Amann A. Chem Soc Rev, 2014, 43: 1423-1449

3 Vishinkin R, Haick H. Small, 2015, 11: 6142-6164 
4 Xu H, Xiang JX, Lu YF, Zhang MK, Li JJ, Gao BB, Zhao YJ, Gu ZZ. ACS Appl Mater Interfaces, 2018, 10: 11785-11793

5 Xu H, Cao KD, Ding HB, Zhong QF, Gu HC, Xie ZY, Zhao YJ, Gu ZZ. ACS Appl Mater Interfaces, 2012, 4: 6752-6757

6 Xu H, Zhang M, Ding H, Xie Z. Microchim Acta, 2013, 180: 85-91

7 Bai L, Xie Z, Cao K, Zhao Y, Xu H, Zhu C, Mu Z, Zhong Q, Gu Z. Nanoscale, 2014, 6: 5680-5685

8 Qian X, Städler B. Chem Mater, 2019, 31: 1196-1222

9 Chen X, Zhou G, Peng X, Yoon J. Chem Soc Rev, 2012, 41: 4610-4630

10 Jelinek R, Ritenberg M. RSC Adv, 2013, 3: 21192-21201

11 Choi JM, Yoon B, Choi K, Seol ML, Kim JM, Choi YK. Macromol Chem Phys, 2012, 213: 610-616

12 Lu Y, Yang Y, Sellinger A, Lu M, Huang J, Fan H, Haddad R, Lopez G, Burns AR, Sasaki DY, Shelnutt J, Brinker CJ. Nature, 2001, 410: 913917

13 Kauffman JS, Ellerbrock BM, Stevens KA, Brown PJ, Pennington WT, Hanks TW. ACS Appl Mater Interfaces, 2009, 1: 1287-1291

Dolai S, Bhunia SK, Beglaryan SS, Kolusheva S, Zeiri L, Jelinek R. ACS Appl Mater Interfaces, 2017, 9: 2891-2898

5 Tu MC, Cheema JA, Yildiz UH, Palaniappan A, Liedberg B. J Mater Chem C, 2017, 5: 1803-1809

Wang X, Sun X, Hu PA, Zhang J, Wang L, Feng W, Lei S, Yang B, Cao W. Adv Funct Mater, 2013, 23: 6044-6050

17 Wang T, Guo Y, Wan P, Sun X, Zhang H, Yu Z, Chen X. Nanoscale, 2017, 9: 869-874

18 Eaidkong T, Mungkarndee R, Phollookin C, Tumcharern G, Sukwattanasinitt M, Wacharasindhu S. J Mater Chem, 2012, 22: 5970

19 Davis BW, Burris AJ, Niamnont N, Hare CD, Chen CY, Sukwattanasinitt M, Cheng Q. Langmuir, 2014, 30: 9616-9622

20 Wang X, Gu Y, Xiong Z, Cui Z, Zhang T. Adv Mater, 2014, 26: 1336-1342

21 Yang J. China Syn Resin Plastics, 1991, 4: 39-43

22 Righettoni M, Tricoli A, Pratsinis SE. Anal Chem, 2010, 82: 3581-3587

23 Wang L, Teleki A, Pratsinis SE, Gouma PI. Chem Mater, 2008, 20: 4794-4796

24 Jang JS, Choi SJ, Kim SJ, Hakim M, Kim ID. Adv Funct Mater, 2016, 26: 4740-4748

25 Güntner AT, Righettoni M, Pratsinis SE. Sens Actuat B-Chem, 2016, 223: 266-273 


\title{
Flexible PDA film array for dual-mode optical sensing of VOC biomarker vapor
}

\author{
Hua $\mathrm{Xu}^{*}$, Yuan An, Mingkun Zhang, Jiajia Li, Zhongze Gu \\ State Key Laboratory of Bioelectronics, School of Biological Science and Medical Engineering, Southeast University, Nanjing 210096, China \\ *Corresponding authors (email: huaxu@seu.edu.cn; gu@seu.edu.cn)
}

\begin{abstract}
In this study, polydiacetylenes (PDAs) film based on the oleophilic double-sides tape as substrate was fabricated by simple drop-casting and illumination, which exhibited dramatic fluorescence and colorimetric change in the presence of VOC vapor in 2 min. Four diacetylene monomers with different chemical structure were polymerized on the double-sides tape substrate for a PDA film array, which exhibited a superior response to VOC vapor and eight different VOC vapors could be detected and discriminated using the as-prepared PDA array combined with a pattern recognition approach. Analysis of exhaled breath using these as-prepared PDA array showed that the simulated exhaled breath of diabetic patients and nephrotic patients, and the exhaled breath of healthy individuals could clearly be identified and distinguished, indicating promising application of the PDA array in disease diagnosis. Compared with the reported PDA film array, our PDA array has significant advantages in rapid response, high sensitivity, excellent flexibility, as well as simple, low-cost, and easy to mass-produce characteristics.
\end{abstract}

Keywords: PDA film array, VOC vapors detection, fluorescence, colorimetric, disease diagnosis

doi: $10.1360 /$ SSC-2019-0072 